\title{
IWPAAMS2007-01: Multiagent System For Management And Monitoring Of Surveillance Routes
}

\author{
Dante I. Tapia, Juan F. De Paz, Sara Rodríguez y Juan M. Corchado
}

\begin{abstract}
This paper presents a multiagent system to manage and monitor the surveillance routes of the security personnel in the construction sector. Several concepts, derived from the Artificial Intelligence, have been taken into account for the system design, obtaining context information in order to automatically monitor the surveillance routes in execution time. The system is composed of intelligent agents with reasoning and planning capabilities, as well as of a set of technologies allowing users to interact with the system and the environment in a natural, unobstructive and easy way.
\end{abstract}

Keywords - Agents, Planning, Artificial Neural Networks, Radiofrequency Identification.

\section{INTRODUCCIÓN}

A ctualmente se registra un crecimiento considerable en el desarrollo de tecnologías de automatización [3]. Diversas arquitecturas, basadas en la utilización de agentes han emergido gracias a la aparición de espacios inteligentes y la integración de dispositivos programables a través de redes computacionales [18]. Esto ha impulsando el desarrollo de la computación ubicua [11], la cual es el acercamiento tecnológico más prometedor para resolver el desafío de desarrollar estrategias que permitan la temprana detección y prevención de problemas en entornos automatizados [1]. Los agentes y los sistemas multiagente son cada vez más relevantes en el desarrollo de sistemas dinámicos y distribuidos, adquiriendo gran aceptación en áreas como el comercio electrónico, la medicina, la oceanografía, la

Este trabajo se ha llevado a cabo gracias a los proyectos MCYT TIC200307369-C02-02 y JCYL-2002-05 proyecto SA104A05. Especial agradecimiento a Telefónica Móviles y a Sokymat por el apoyo brindado, así como por la tecnología proporcionada.

Dante I. Tapia, Departamento de Informática y Automática de la Universidad de Salamanca, Plaza de la Merced s/n, 37008, España (correo electrónico: dantetapia@usal.es).

Juan F. De Paz, Departamento de Informática y Automática de la Universidad de Salamanca, Plaza de la Merced s/n, 37008, España (correo electrónico: fcofds@usal.es)

Sara Rodríguez, Departamento de Informática y Automática de la Universidad de Salamanca, Plaza de la Merced s/n, 37008, España (correo electrónico:srg@usal.es)

Juan M. Corchado es Decano de la Facultad de Ciencias y profesor Titular en el Departamento de Informática y Automática en la Universidad de Salamanca (e-mail: corchado@usal.es) robótica, etc. [4].

Los sistemas basados en la Inteligencia Ambiental han ampliado los campos de aplicación práctica a medida que se han desarrollado nuevos contextos de movilidad dentro de la sociedad de la información. Actualmente existe una creciente necesidad de encontrar vías más efectivas para proporcionar servicios en dispositivos móviles [18] como asistentes personales (PDA: Personal Digital Assistant) y teléfonos móviles, empleando tecnologías de comunicación como GPRS (General Packet Radio Service), UMTS (Universal Mobile Telecommunications System), Bluetooth, etc. Por tal motivo, es necesario entender mejor la visión de la Inteligencia Ambiental [5], con la finalidad de obtener los mejores beneficios que este modelo de interacción puede ofrecer.

En este artículo se presenta un sistema multiagente desarrollado con el objetivo de mejorar el control sobre las actividades que desempeña el personal encargado de la vigilancia en las obras en construcción. El sistema se implementa en entornos altamente dinámicos, monitorizando al personal de la obra y gestionando las rutas de vigilancia, de forma automática y en tiempo de ejecución.

El sistema hace uso de un conjunto de tecnologías, como Wi-Fi, GPRS e identificación por radiofrecuencia (RFID: Radio Freqyency IDentification). Estas tecnologías aportan una infraestructura capaz de soportar las necesidades de comunicación distribuida de los agentes. Además, incrementan la movilidad, la flexibilidad y la eficiencia de los usuarios, al permitir que los servicios (programas, datos, equipos, etc.) estén disponibles para cualquier equipo de la red que lo requiera, sin importar la localización física del recurso y del usuario.

A continuación, se presenta la problemática en torno a la monitorización de las actividades de los trabajadores en el sector de la construcción. Posteriormente se presenta un caso de estudio, en donde se describe el desarrollo de un sistema multiagente que ayude a resolver algunos de los problemas que afectan al sector de la construcción. Después, se describen brevemente las principales características del sistema, para finalizar con los resultados y conclusiones obtenidos después de realizar una serie de simulaciones con un prototipo del sistema. 


\section{ProblemÁtica del Sector de la CONSTRUCCión}

En los últimos años se ha presentado un crecimiento considerable en el sector de la construcción sobre todo en los países desarrollados [17]. Por ejemplo, en el año 2001, en Japón el 10\% y en Francia el 5,6\% del total de trabajadores se encontraban en la industria de la construcción [13]. Particularmente en España, en el 2001 esta industria empleaba a 1.850.200 trabajadores, que significa el $11,6 \%$ del total de los trabajadores del país, absorbiendo el $26,4 \%$ de todos los accidentes de trabajo [13][8]. En un sector tan pujante y en creciente desarrollo, se hace cada vez más necesario establecer políticas de seguridad para la administración de riesgos en las obras en construcción, que permitan prever y controlar condiciones, conductas y situaciones conflictivas, contribuyendo de esta manera a la mejora de la calidad de vida de los trabajadores y el incremento de la productividad. Según recientes estudios [7], un 3\% de la jornada laboral se pierde por falta de sistemas de control de tiempos que permitan verificar la duración real del tiempo trabajado. La implementación de sistemas de control amplía la productividad, ya que el trabajador optimiza su potencial y agiliza los procesos en que interviene [3].

Por lo tanto, es necesario desarrollar métodos que permitan llevar un mejor control sobre los trabajadores durante el proceso de construcción de las obras [16]. Las incidencias derivadas de la incorrecta supervisión del personal, en especial los robos de material, el acceso de personas no autorizadas, así como la inadecuada vigilancia de las instalaciones, son problemas comunes que aumentan los riesgos y afectan directamente a todo el proceso. Los sistemas de tele-presencia y vigilancia remota son cada vez más comunes en este tipo de escenarios, permitiendo a los supervisores observar a distancia el comportamiento de los trabajadores y el estado de las instalaciones.

Los sistemas multiagente y las arquitecturas basadas en dispositivos inteligentes han sido recientemente explorados como sistemas de supervisión y seguimiento, siendo capaces de implementarse en gran cantidad de escenarios, incluido el sector de la construcción [19][24]. Este tipo de sistemas son adecuados para mejorar la gestión de sistemas complejos, especialmente aquellos que integran aspectos físicos, sociales o económicos, entre ellos los aplicados en la construcción. Los sistemas multiagente ofrecen robustez, alta tolerancia a fallos, escalabilidad, y coordinación entre sus componentes [9], características que permiten aplicarlos en gran variedad de escenarios, por ejemplo, en el control de sistemas de fabricación [6][15].

A continuación, se presenta un caso de estudio, en el cual se hace uso de diversas tecnologías y mecanismos que permiten programar la jornada laboral del personal de seguridad dentro de las obras en construcción, y a su vez, monitorizar su trabajo de manera automática y en tiempo de ejecución.

\section{CASO DE Estudio}

Con la finalidad de proporcionar un mejor control sobre las actividades que desempeña el personal encargado de vigilar las obras en construcción, se ha diseñado un sistema que permite planificar y distribuir las rutas entre el personal disponible, y a su vez, monitorizar el cumplimiento de las rutas asignadas, todo ello de forma automática y en tiempo de ejecución.

El sistema interactúa con los usuarios y el entorno a través de un conjunto de agentes inteligentes, asistentes personales (PDA), redes inalámbricas Wi-Fi (Wireless Fidelity), tecnología RFID y tecnología GPRS (General Packet Radio Service), trabajando de forma conjunta para proporcionar informes, respuestas, planes, etc. en tiempo de ejecución a los usuarios. Las redes Wi-Fi pueden ser sustituidas por GPRS dependiendo de las dimensiones y características de cada obra en construcción.

Los agentes realizan una planificación de las rutas de vigilancia según el personal disponible para cubrir toda la superficie de la obra. El sistema tiene la capacidad de replanificar las rutas de forma automática, dependiendo del personal disponible en cada momento o incluso al informar al sistema de pasos cortados por motivo de los mismos avances de las obras de construcción. El supervisor es capaz de monitorizar en cada momento, vía Internet, la actividad del personal de seguridad en cada unas de las obras asignadas.

La información del contexto, la cual sirve para detectar la posición del personal de seguridad dentro de las obras, es almacenada en un ordenador central y después analizada para detectar posibles irregularidades en las actividades del personal, por ejemplo un seguimiento incorrecto, incompleto, o incluso omitido de una determinada ruta de vigilancia asignada. La posición del personal se obtiene a través de "puntos de control", haciendo uso de un conjunto de tecnologías para detectar e identificar al personal que ha pasado por dichos puntos.

La identificación por radiofrecuencia (RFID) es clave para la adecuada monitorización de las rutas asignadas. RFID es una tecnología para la captura automática de datos que puede ser usada para identificar electrónicamente y guardar información de productos, artículos, componentes, e incluso personas [21]. Un sistema RFID se compone principalmente de cuatro elementos [20]: 1) Etiquetas, 2) Lectores, 3) Antenas y Radios y 4) Hardware de Procesamiento. Los chips RFID son leídos a través de un campo electromagnético que opera en las frecuencias entre $100 \mathrm{KHz}$ y $15 \mathrm{MHz}$, induciendo una corriente eléctrica en la cola del transponders (etiqueta sin sistema de energía incorporado), la cual energiza al chip y éste a su vez transmite una respuesta de baja intensidad y con una frecuencia diferente que es detectada por el lector, quien la envía finalmente a un ordenador o a un dispositivo que muestre la información del chip [20]. Como se muestra en la Fig. 1, la configuración utilizada en este desarrollo consiste en una serie de transponders colocados en diferentes puntos estratégicos (puntos de control) de la obra, PDAs con lectores RFID incorporados y un ordenador central en donde es procesada y almacenada la información, la cual puede ser consultada vía Internet. El número de transponders instalados 
depende de los puntos de control requeridos para cada obra.

El personal de seguridad, al hacer la ronda de la ruta de vigilancia determinada por el sistema, debe pasar por cada uno de los puntos de control asignados. Al llegar a cada punto de control, debe acercar la PDA para leer el identificador del transponder colocado en dicho punto. La información es obtenida y enviada automáticamente vía Wi-Fi o GPRS al ordenador central, en donde se almacena la hora, el identificador del punto de control y el identificador del trabajador asociado con la PDA. Cuando un trabajador no registra el paso por un determinado punto de control, el sistema automáticamente envía un SMS (Short Message Service) o un e-mail al supervisor, además de almacenar la incidencia en la base de datos.

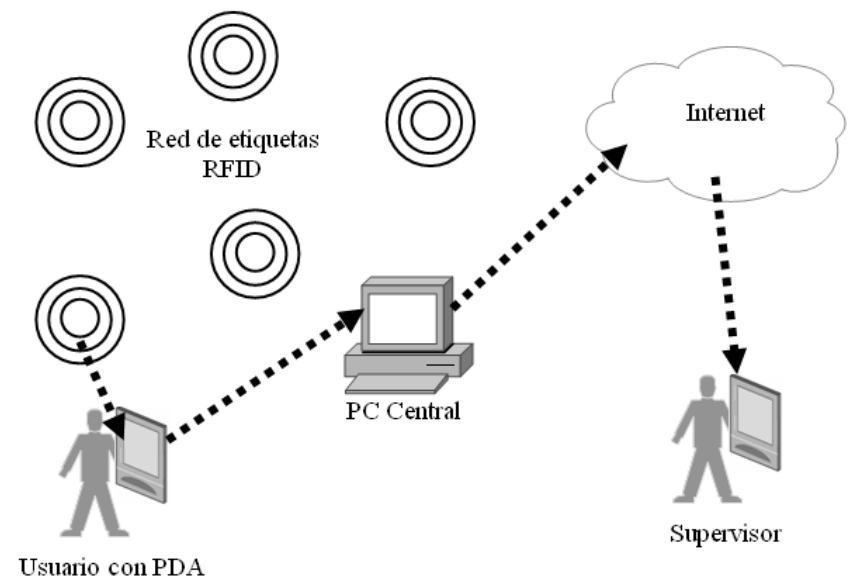

Fig. 1. Esquema básico de monitorización

\section{ARquitectura DEL Sistema}

Los sistemas multiagente son sistemas distribuidos basados en la cooperación entre agentes autónomos [3]. Las arquitecturas multiagente distribuidas presentan una buena capacidad de recuperación ante errores sin necesidad de tener que reiniciar todo el sistema [22].

El diseño y modelado del sistema se ha realizado mediante una combinación de las metodologías para el desarrollo de sistemas multiagente Gaia [23] y AUML [2]. Gaia es una metodología sencilla y con un alto nivel de abstracción para el análisis y diseño de sistemas multiagente [23]. Tiene como objetivo proporcionar un análisis del sistema y diseñar su estructura a partir de un conjunto de requisitos iniciales, obteniendo un conjunto de modelos que permiten definir y analizar la estructura básica del sistema [23]. Los modelos conseguidos con Gaia se refinan y adaptan empleando AUML, obteniendo una serie de diagramas que permiten obtener un modelo del sistema más cercano a la implementación [2].

Una vez definido y obtenido el modelo del sistema, como se muestra en la Fig. 2, se aprecia que el sistema se compone de cinco tipos de agentes:

- Agente Vigilante. Está asociado a cada PDA y se encarga de realizar la lectura de los transponders en cada punto de control. Se comunica con el Agente Controlador para comprobar que se cumple correctamente la ruta asignada a cada vigilante de seguridad. Existe un agente de este tipo por cada vigilante de seguridad, ejecutándose en la correspondiente PDA. Este agente se comunica constantemente con el Agente Controlador para obtener las rutas planificadas y, posteriormente, se la comunica al vigilante correspondiente. Además, envía las lecturas obtenidas de los puntos de control al Agente Controlador, para que realice las acciones pertinentes. La interfaz de usuario del Agente Vigilante permite introducir incidencias que son enviadas al Agente Manager.

- Agente Manager. Realiza el control general del sistema. Controla la conexión y desconexión al sistema de los Agentes Vigilante e informa de ello al Agente Planificador para que calcule las rutas de vigilancia en la obra. Obtiene las rutas del Agente Planificador e informa a los Agentes Controlador el tiempo estimado en que el personal de seguridad debe pasar por los puntos de control. Recibe las incidencias de los Agentes Controlador y Vigilante y, dependiendo de su prioridad, informan de las mismas al Agente Móvil. En caso de que la incidencia se reciba de un Agente Controlador, se comunica la incidencia al Agente Vigilante para que el usuario introduzca una descripción del evento. El Agente Manager almacena toda la información generada por el sistema (incidencias, fecha, hora, puntos de control fichados y omitidos, etc.) en la base de datos. La información puede ser consultada vía Internet, desde un navegador Web. Este agente, además, se encarga de la decisión del envío de alertas a los supervisores, vía Wi-Fi, SMS o GPRS.

- Agente Planificador. Se encarga de generar automática y dinámicamente las rutas de vigilancia para el personal de seguridad. Las rutas generadas son enviadas al Agente Manager para que éste las envíe al personal correspondiente. El mecanismo de planificación de rutas se describe en la siguiente sección.

- Agente Controlador. Realiza una monitorización de los puntos de control por los que el personal de seguridad debe pasar. Una vez obtenida la ruta generada por el Agente Planificador, se realiza un cálculo del tiempo necesario para completar cada punto de control. Con la ruta y el cálculo del tiempo total de la ruta, se informa al Agente Manager y al Agente Vigilante acerca de la ruta y de las veces que debe completar el trayecto. El Agente Controlador realiza un seguimiento de las incidencias que se presenten durante la ejecución de la ruta por cada vigilante de seguridad, y las envía al Agente Manager.

- Agente Móvil. Se encarga del envío de mensajes a los supervisores. Recibe del Agente Manager las incidencias y las envía vía Wi-Fi, SMS o GPRS. En caso de que se produzcan errores en la conexión o el envío del mensaje, comunica dicha incidencia al Agente Manager para que registre dichos errores en la base de datos.

La comunicación entre los Agentes Vigilante, ejecutados en PDA, y el resto de agentes del sistema se realiza utilizando 
Wi-Fi o GPRS, dependiendo de las necesidades de cada obra.

A continuación, se describe brevemente el mecanismo para la planificación de rutas, detallando las características principales que permiten generar dinámicamente rutas para el personal de seguridad, de manera automática y en tiempo de ejecución.

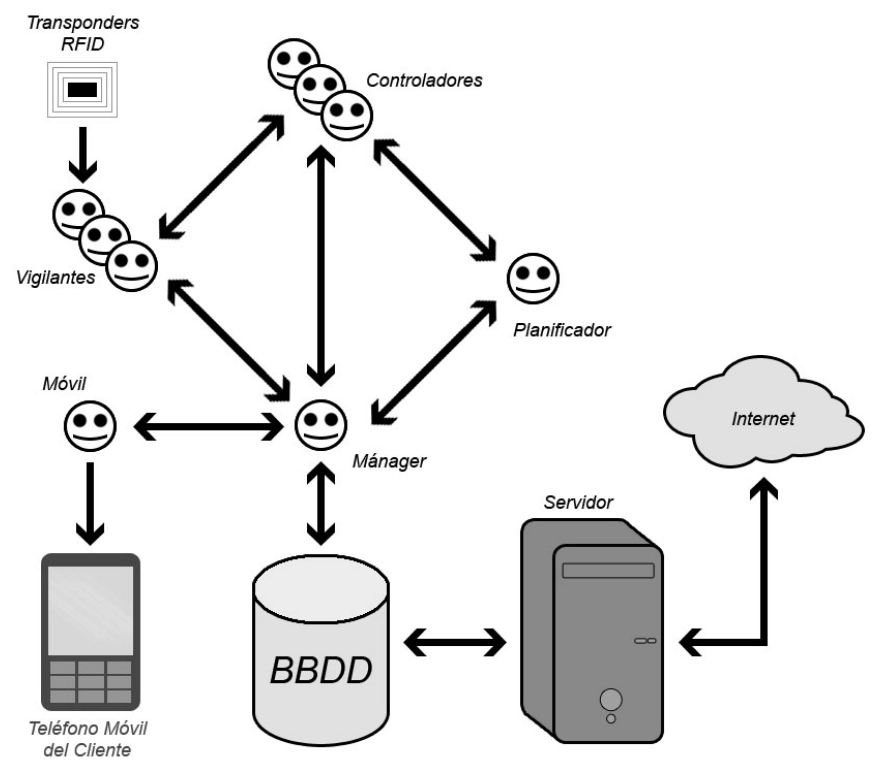

Fig. 2. Estructura del sistema

\section{Mecanismo para la Planificación de Rutas de VIGILANCIA}

Para el cálculo de las rutas, el sistema considera la minimización del recorrido, por lo tanto, será necesaria una adecuada agrupación de los puntos a recorrer, así como un buen establecimiento del orden a seguir dentro de cada grupo. En particular, el agente Planificador, será el encargado de realizar la planificación de rutas, repartiendo estos puntos de control entre el personal de seguridad mediante una red de Kohonen de tipo SOM (Self Organizing Maps), utilizando el algoritmo de aprendizaje de las k-medias [12]. Una vez repartidos los puntos de control, se envía la distribución de los puntos a cada agente Controlador, el cual calcula la ruta a seguir por cada vigilante de seguridad. El agente Controlador emplea una modificación de la red neuronal SOM para calcular la ruta a seguir por cada vigilante.

Se realiza una modificación del mapa autoorganizado SOM mediante una Red Neuronal FYDPS, modificando la función de vecindad definida en la etapa de aprendizaje de la red de Kohonen. La red está formada por dos capas, la de entrada y la de salida. En la capa de entrada se tienen dos neuronas, correspondientes a las coordenadas de los puntos de control a visitar por el vigilante. En la capa de salida, existen tantas neuronas como puntos de control haya que visitar, una vez realizada la división de puntos de control [10][14].

Sean $x_{i} \equiv\left(x_{i 1}, x_{i 2}\right) \quad i=1, \cdots . N$ las coordenadas del punto de control $i$ y $n_{i} \equiv\left(n_{i 1}, n_{i 2}\right) \quad i=1, \cdots, N \quad$ las coordenadas de las neuronas i en $\mathfrak{R}^{2}$, siendo $N$ el número de puntos de control a visitar por un vigilante. Habrá dos neuronas en la capa de entrada y $N$ neuronas en la capa de salida. La fórmula de actualización de los pesos viene definida por la siguiente ecuación:

$$
w_{k i}(t+1)=w_{k i}(t)+\eta(t) g(k, h, t)\left(x_{i}(t)-w_{k i}(t)\right)
$$

Siendo $w_{k i}$ el peso que conecta la neurona $i$ de la capa de entrada con la neurona $k$ de la capa de salida. $t$ representa la iteración, $\eta(t)$ la tasa de aprendizaje y finalmente la función de vecindad $g(k, h, t)$ que depende de tres parámetros, la neurona ganadora, la neurona actual y la iteración.

Se considerará una función de vecindad decreciente con el número de interacciones y con la distancia a la neurona ganadora.

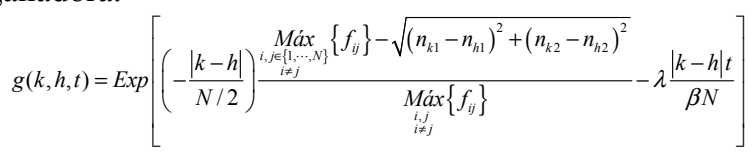

$\lambda$ y $\beta$ se determinan empíricamente. El valor de $\lambda$ por defecto se establece a 1 y el de $\beta$ a valores comprendidos entre 5 y $50 . t$ es la iteración actual, su valor máximo viene definido por $\beta N . \operatorname{Exp}[x]=e^{x} . N$ es el número de puntos de control a visitar por un vigilante fijo, $f_{i j}$ es la distancia entre los puntos $i$ y $j$. Finalmente, $\operatorname{Max}\left\{f_{i j}\right\}$ representa la máxima distancia que une cualquiera de los dos puntos a recorrer del grafo. Cada uno de los tres términos de la función de vecindad se definen de modo que su valor se encuentre entre $(0,1)$. El radio de la función de vecindad al final del aprendizaje debe ser próximo a 0 para que se actualice solamente el ganador.

En el entrenamiento de la RNA, el grupo de puntos de control se pasa a las neuronas de la capa de entrada, de forma que los pesos de las neuronas se acerquen a las coordenadas de los puntos de control. Cuando concluye el proceso, existe una neurona asociada a cada punto de control. Para determinar la ruta a seguir, se une el punto de control asociado a la neurona $i$ con el asociado a la $i+1$, con $i=1,2, \ldots, N$, pasando por todo el vector de neuronas. Para finalizar la ruta, se agrega un último tramo que una el punto de control en la obra asociado a la neurona $N$ con el asociado a la neurona 1 . La distancia del camino se calculará mediante la suma de distancias entre las sucesivas parejas de puntos de control de dicho camino. La tasa de aprendizaje empleada se define de forma decreciente y depende del número de iteraciones $t$ tal y como se indica a continuación.

$$
\eta(t)=\operatorname{Exp}\left[-\sqrt[4]{\frac{t}{\beta N}}\right]
$$

La función de activación y de salida de las neuronas es la identidad. Cuando se finaliza la etapa de aprendizaje, se determina la neurona ganadora para cada punto de control de modo que cada punto de control tiene asociada una única neurona. La ruta a seguir se calcula siguiendo las sucesivas neuronas del vector de pesos sabiendo, tal y como se ha 
definido anteriormente, que cada una se encuentra asociada con su correspondiente punto de control una vez finalizado el aprendizaje. De este modo, el vector define un anillo, donde la neurona $n_{1}$ es la siguiente a la neurona $n_{N}$.

Tomando un radio de vecindad grande inicialmente, las modificaciones de los pesos afectan en mayor medida a las neuronas vecinas, mientras que a medida que el radio de vecindad decrece con las iteraciones, las actualizaciones de las neuronas vecinas decrecen hasta llegar al punto en que sólo afecten a la neurona ganadora. La detención del aprendizaje de la red se produce cuando la distancia entre los puntos de control no se puede optimizar más. El número total inicial de iteraciones es de $T_{1}=\beta N$ (primera fase). Cuando $t=\beta N$, se intercambian las posibles parejas de neuronas (intercambian sus pesos) del anillo de neuronas obtenido, si con ello la distancia se optimiza, en caso de optimización, se reduce el número de iteraciones actuales para seguir con el aprendizaje.

En general, en la fase $Z$, el número total de iteraciones a realizar es:

$$
T_{Z}=T_{Z-1}-\frac{T_{Z-1}}{Z}
$$

El objetivo de estas fases es el de eliminar los cruces. Una vez concluidas las iteraciones de cada fase se comprueba si la distancia está optimizada mediante el intercambio de parejas, deteniéndose el aprendizaje cuando no es posible realizar ningún intercambio de modo que se reduzca la longitud del tamaño recorrido. Por lo tanto, el retroceso en el número de las iteraciones es cada vez menor, de este modo se consigue establecer un número máximo de iteraciones, aunque su valor sea variable.

En la Fig. 3 se observan las rutas planificadas por el agente Controlador para uno y dos vigilantes de seguridad, con la agrupación de puntos de control realizada por parte del agente Planificador con la red SOM.

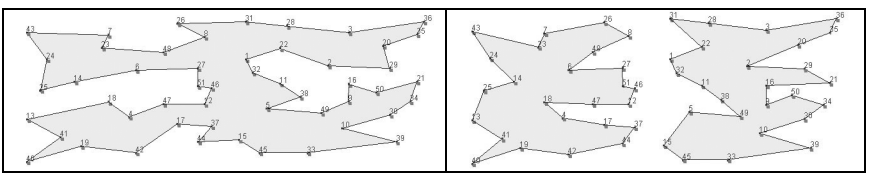

Fig. 3. Izquierda: Ruta calculada para un único vigilante de seguridad; Derecha: Ruta calculada para dos vigilantes de seguridad

\section{CONCLUSIONES}

El sistema desarrollado ha sido probado en entornos controlados, realizando una serie de simulaciones en el cálculo de rutas. Los principales datos de entrada con los que se ha alimentado el sistema son: 1) El número de puntos de control en la obra (por los cuales deben pasar los vigilantes); 2) La distancia entre los puntos de control; 3) El tiempo promedio para ir de un punto de control a otro; 4) El número de vigilantes disponibles para calcular las rutas. En las pruebas realizadas se ha podido observar que es posible determinar si es necesario aumentar o disminuir el personal de seguridad para vigilar una obra, dependiendo de las rutas calculadas. Por ejemplo, la Fig. 4 muestra que la ruta calculada para un solo guardia de seguridad es relativamente grande para una jornada laboral de 8 horas y sólo se puede recorrer la ruta una vez. Al añadir un nuevo guardia de seguridad, las nuevas rutas, mostradas en la Fig. 5 se pueden completar más de 2 veces y media en 8 horas.

Aunque el sistema se encuentra aún en desarrollo, los resultados obtenidos hasta el momento reflejan que es posible optimizar la jornada laboral de los guardias de seguridad, así como maximizar los recursos disponibles para cada obra, ya que a través del sistema es posible determinar el personal necesario para cubrir una determinada área de vigilancia. Además, el sistema proporciona a los supervisores, la capacidad para monitorizar las actividades del personal, detectando cualquier irregularidad en el recorrido de las rutas por parte del personal de seguridad.

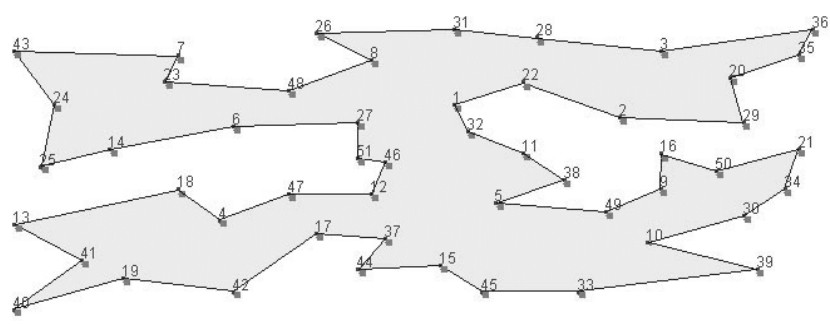

Fig. 4. Ruta calculada para un solo guardia de seguridad (413 min.)
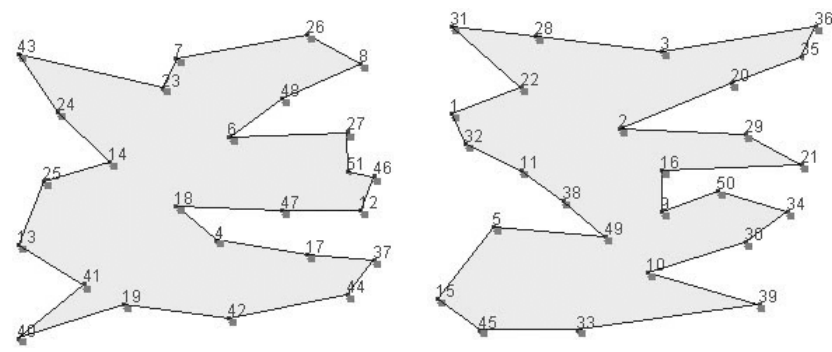

Fig. 5. Rutas calculadas para dos guardias de seguridad (186 y 179 min. respectivamente)

Los sistemas multiagente ofrecen flexibilidad, agilidad, escalabilidad y robustez [9], características necesarias para aplicaciones que deben implementarse en entornos altamente dinámicos y delicados como lo es la industria de la construcción.

El uso de tecnologías como Wi-Fi, RFID o GPRS, permiten obtener información del contexto y reaccionar de forma automática y en tiempo de ejecución, de forma ubicua, natural y sencilla para los usuarios, acercándose cada vez más a la visión de la Inteligencia Ambiental [5], una reciente área multidisciplinar basada en la computación ubicua.

Es necesario que las nuevas tecnologías se adapten a las necesidades de los usuarios, tanto para mejorar la calidad de trabajo como para lograr desarrollos tecnológicos y de gestión que supongan ventajas competitivas diferenciales. El sistema presentado posee la ventaja de ser fácilmente adaptable a otros posibles campos o líneas de aplicación futuras de características similares. 


\section{REFERENCIAS}

[1] C. Angulo y R. Tellez, "Distributed Intelligence for smart home appliances," Tendencias de la minería de datos en España, Red Española de Minería de Datos, 2004.

[2] B. Bauer y M. P. Huget, "FIPA modeling: agent class diagrams," Working Draft, foundation for Intelligent Physical Agents. Disponible en: http://www.auml.org/

[3] N. Carretero y A. B. Bermejo, "Inteligencia Ambiental," CEDITEC: Centro de Difusión de Tecnologías, Universidad Politécnica de Madrid, España, 2005.

[4] J. M. Corchado, J. Bajo, Y. De Paz y D. I. Tapia, "Intelligent Environment for Monitoring Alzheimer Patients, Agent Technology for Health Care," Decision Support Systems, Eslevier, Amsterdam, Netherlands, 2007, In press.

[5] P. L. Emiliani y C. Stephanidis, "Universal access to ambient intelligence environments: opportunities and challenges for people with disabilities," IBM Systems Journal, 2005.

[6] T. Heikkilä, M. Kollingbaum, P. Valckenaers y G. J. Bluemink, "An Agent Architecture for Manufacturing Control: manAge," Computers in Industry, no. 46, pp. 315-331, 2001

[7] Inology, Nota de prensa, 9 de Junio de 2005. Disponible en: http://www.controldetiempos.com/sala de prensa.htm\#absentismo

[8] Ministerio de Trabajo y Asuntos Sociales, "Estadísticas de Accidentes de Trabajo," Disponible en http://www.mtas.es/insht/statistics/mtas.htm

[9] N. R. Jennings y M. Wooldridge, "Applications of intelligent agents," In Agent Technology: Foundations, Applications, and Markets, N. R. Jennings and M. J. Wooldridge, Eds. Springer-Verlag New York, Secaucus, NJ, 3-28, 1998.

[10] H. D. Jin, K. S Leung, M. L. Wong y Z. B. Xu, "An Efficient SelfOrganizing Map Designed by Genetic Algorithms for the Traveling Salesman Problem," IEEE Transactions on Systems, Man, and Cybernetics, Part B: Cybernetics, vol. 33, no. 6. pp. 877-888, 2003

[11] J. Kleindienst, T. Macek, L. Seredi, y J. Sedivy, "Vision-enhanced multimodal interactions in domotic environments". IBM Tecnologías de Voz y Sistemas, República Checa, 2004.

[12] T. Kohonen, "Self-Organizing Maps," Springer-Verlag, New York, Inc., 1997.

[13] LABORSTA, ILO on-line database on labour statistic, International Labour Organization, 2007. Disponible en: http://laborsta.ilo.org

[14] K. S. Leung, H. D. Jin, y Z. B. Xu, "An expanding Self-organizing Neural Network for the Traveling Salesman Problem," Neurocomputing, vol. 62 , pp. 267-292, 2004

[15] M. K. Lim y Z. Zhang, "A Multi-agent Based Manufacturing Control," Strategy for Responsive manufacturing. Journal of Materials Processing Technology, 2003.

[16] J. Parada-Pardo, "El proceso de diversificación en el sector de la construcción," Directivos Construcción, no. 147, pp. 38, 2002.

[17] P. Lleonard y A. Garola, "Estrategias empresariales en el sector de la construcción," Gabinet Estudis Econòmics, Barcelona, 2001.

[18] P. Rigole, T. Holvoet y Y. Berbers, "Using Jini to Integrate Home Automation in a Distributed Software-System". In Revised Papers From the 4th international Workshop on Distributed Communities on the Web (April 03 - 05, 2002). J. Plaice, P. G. Kropf, P. Schulthess, and J. Slonim, Eds. Lecture Notes In Computer Science, vol. 2468. SpringerVerlag, London, 291-304.

[19] J. A. Araúzo, J. J. de Benito Martín y R. Del Olmo Martínez, "El control de planta: una aproximación basada en agentes," $V$ Congreso de Ingeniería de Organización, Valladolid-Burgos, 2003.

[20] S. Garfinkel y B. Rosenberg, "RFID: Applications, security, and privacy," Addison-Wesley Professional, pp. 15-36, 2005.

[21] U.S. Department of Commerce, "Radio Frequency Identification," Opportunities and Challenges in Implementation, Washington, D.C., 2005.

[22] M. Wooldridge y N. R. Jennings, "Agent Theories, Architectures, and Languages," a Survey, Wooldridge \& Jennings ed., Intelligent Agents, Springer-Verlag, pp. 1-22, 1995.

[23] M. Wooldridge, N. R. Jennings y D. Kinny, "The Gaia Methodology for Agent-Oriented Analysis and Design". Journal of Autonomous Agents and Multi-Agent Systems, 3 (3), pp. 285-312, 2000.

[24] A. López Paredes y J. M. Galán Ordax, "Gestión del agua doméstica en la Región Metropolitana de Barcelona: Una aproximación participativa, basada en agentes," $V$ Congreso de Ingeniería de Organización, Valladolid-Burgos, pp. 169-170, 2003.

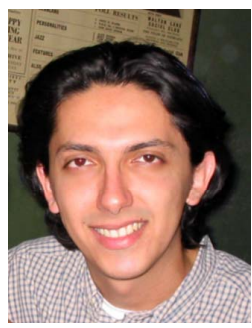

Dante I. Tapia. En 2009 recibió el grado de Doctor en Informática y Automática por la Universidad de Salamanca, España, bajo la supervisión del Dr. Juan M. Corchado. Obtuvo los títulos de Ingeniero en Sistemas Computacionales y el Master en Telemática en la Universidad de Colima, México, en los años 2001 y 2004 respectivamente. Ha colaborando en la gestión de proyectos $\mathrm{I}+\mathrm{D}+\mathrm{i}$ con el Gobierno del Estado de Colima (México), la Universidad de Colima (México), y el grupo BISITE (Biomedicina, Sistemas Inteligentes y Tecnología Educativa) de la Universidad de Salamanca (España). Es autor de diversos artículos publicados en reconocidas revistas, congresos y simposios internacionales.

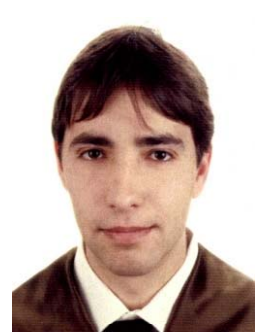

Juan F. De Paz. En la actualidad está realizando los estudios de Doctorado de Informática y Automática en la Universidad de Salamanca, España, bajo la supervisión del Dr. Juan M. Corchado. En 2007 el Ministerio de Educación y Ciencia le otorgó una beca de Formación del Personal Investigador (FPI) y participa en diversos proyectos de I+D. Obtuvo por la Universidad de Salamanca los títulos de Ingeniería Técnica en Informática de Sistemas en el año 2003, Ingeniería Informática en 2005 y Diplomatura de Estadística en 2007. Es autor de artículos publicados en reconocidas revistas, congresos y simposios internacionales.

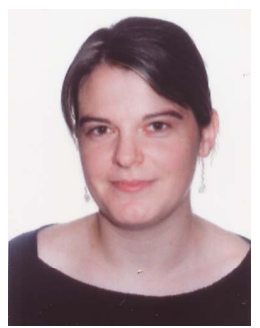

Sara Rodríguez. Actualmente cursa los estudios de Doctorado en Informática y Automática, en la Universidad de Salamanca, España, bajo la supervisión del Dr. Juan M. Corchado. Está vinculada a proyectos de investigación dentro del grupo BISITE (Biomedicina, Sistemas Inteligentes y Tecnología Educativa) de la Universidad de Salamanca gracias a un contrato de personal investigador desde principios del año 2007. Obtuvo el título de Ingeniería Técnica en Informática de Sistemas en el año 2004 y el título de Ingeniera Informática en mayo de 2007 por la Universidad de Salamanca (España). Ha participado como coautora en artículos publicados en reconocidos congresos y simposios internacionales.

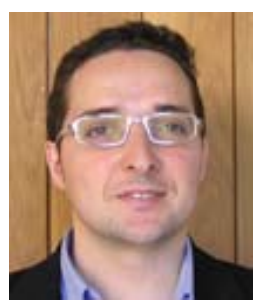

Juan M. Corchado Actualmente es Pprofesor Titular en la Universidad de Salamanca (España). Obtuvo un doctorado en Ciencias de la Computación por la Universidad de Salamanca en 1998 y un doctorado en Inteligencia Artificial por la Universidad de Paisley (Glasgow, Reino Unido) en 2000. Es Director del grupo de Bioinformatica, Sistemas Inteligentes y Tecnología Educativa (http://bisite.usal.es) y Director del Máster en Comercio Electrónico de la Universidad de Salamanca (España). Anteriormente fue sub-director de la Escuela de Informática de la Universidad de Vigo (España) (1999-00) e investigador en la Universidad de Paisley (Reino Unido, 1995-98). Ha sido investigador colaborador en el Plymouth Marine Laboratory (Reino Unido) desde 1993. Ha liderado múltiples proyectos de investigación financiados por diversas instituciones españolas y europeas, públicas y privadas, $\mathrm{y}$ ha dirigido ocho tesis doctorales. Es co-autor de aproximadamente 150 libros, capítulos de libros, artículos en revistas internacionales, etc. publicados por organizaciones tales como Elsevier, IEEE, IEE, ACM, AAAI, Springer Verlag, Morgan Kaufmann, etc., y la mayor parte de ellos presentan investigaciones teóricas y prácticas en el campo de la Inteligencia Artificial y los sistemas distribuidos. Ha sido Presidente de numerosos comités organizadores y científicos en distintos simposios. 